\title{
FREE-ENERGY FUNCTIONS FOR ELASTIC-PLASTIC MATERIAL ELEMENTS
}

\author{
BY \\ MASSIMILIANO LUCCHESI \\ CNUCE - Istituto del C.N.R. via S. Maria, 36 PISA
}

\begin{abstract}
Certain thermodynamic properties of elastic-plastic materials with workhardening are discussed and their corresponding free-energy functions are determined.
\end{abstract}

1. Introduction. The aim of the present paper is to examine certain thermodynamic properties of elastic-plastic materials and to determine their free-energy functions. We shall confine ourselves to considering the von Mises yield criterion, infinitesimal deformations, and isothermal conditions.

It is shown in [1] how, under suitable hypotheses on the spatial gradient of velocity, the infinitesimal theory of plasticity can be deduced from the general theory of materials with elastic range, a theory formulated in [2] and [3].

In order to describe the constitutive response, we use the concepts of material element, state, and process (the latter is a mapping defined on a real interval, which takes its values in the set of states and specifies the possible evolution from an initial state). We formulate these concepts as in Šilhavý [4, 5].

The following general properties of material elements, states, and processes are important to our developments. If a material element satisfies the condition of perfect accessibility (i.e., if any two states are linked by at least one process), then the second law of thermodynamics states [6] that the work done by the exterior on the material element during a closed process is nonnegative. If the second law is satisfied, at least one state function $\psi$ exists, called free-energy function, which satisfies the dissipation inequality, i.e., the inequality saying that the work done during any process linking two states $\sigma_{1}$ and $\sigma_{2}$ is not less than $\left(\psi\left(\sigma_{2}\right)-\psi\left(\sigma_{1}\right)\right)$. In general, the function $\psi$ is not unique but, for each state $\sigma$, the set of all free-energy functions vanishing at $\sigma_{0}$ has least and upper elements, i.e., free-energy functions $\psi$ and $\bar{\psi}$ such that $\underline{\psi} \geq \psi \geq \bar{\psi}$, for any free-energy function $\psi$ with $\psi\left(\sigma_{0}\right)=0$.

If a material element does not meet the condition of perfect accessibility but has a base state, i.e., a state from which every other state is reachable by some process, it is still possible to formulate the second law in such a way that its prescription is equivalent to the existence of (at least) one free-energy function [7].

Received June 19, 1991 and, in revised form, August 5, 1991.

(C)1993 Brown University 
Here we first consider materials with combined (i.e., kinematic and isotropic) work-hardening. A state is a triplet $\sigma=\left(E, E^{p}, \zeta\right)$ consisting of the infinitesimal strain $E$, plastic strain $E^{p}$, and Odqvist parameter $\zeta$; a process is an evolution mapping $\tau \rightarrow \sigma(\tau)=\left(E(\tau), E^{p}(\tau), \zeta(\tau)\right)$. For each state $\sigma$, the corresponding Odqvist parameter is interpreted as the length of the plastic strain path during any process beginning at one and the same state $\sigma_{0}$ and ending in $\sigma ; \zeta$ determines the radius of the elastic range associated with $\sigma$. The state $\sigma_{0}$ has the role of basic state; at $\sigma_{0}$, all of the infinitesimal strain, the plastic strain, and the Odqvist parameter are null, and we interpret $\sigma_{0}$ as the annealed state of the material. Thus, the material element we construct for materials with combined work-hardening has a base state; however, it does not satisfy the perfect accessibility condition, because $\zeta$ cannot diminish during any process.

For each state $\sigma$ we calculate the work done along any process beginning in $\sigma_{0}$ and ending in $\sigma$. In this way, we check that the work depends solely on $\sigma$, so that there exists a free-energy function that vanishes in $\sigma_{0}$ and verifies the dissipation inequality as an equality. This fact is at first sight surprising, especially if we consider that, as we prove, the material element does not contain superfluous state variables. We also determine all the free-energy functions corresponding to the element.

Secondly, by way of comparison, we construct the material element for materials with kinematic work-hardening. In this case the radius of the elastic range is a material constant and it is, therefore, not necessary to include the Odqvist parameter among the state variables: the state is identified by the corresponding strain and plastic strain alone. For this reason, the material element with kinematic workhardening is not a special case of a material element with combined work-hardening. It will be shown that a material element with kinematic work-hardening satisfies the condition of perfect accessibility and all its free-energy functions will be determined. The work done along any process that begins in the annealed state and ends in $\sigma$ depends, as in the previous case, upon the length of the plastic strain path and cannot now be expressed only as a function of the state variables. Thus, in this case no freeenergy function satisfies the dissipation inequality as an equality.

2. Thermodynamic background. This section, taken from Šilhavý [8], is devoted to a brief presentation of the material element and of certain restrictions imposed by the second law of thermodynamics on the constitutive response of the material in isothermal conditions.

2.1. Material element. In this subsection, we introduce the concept of a material element, following the scheme to be found in $[4,5]$. We shall confine ourselves to examining the case of isothermal infinitesimal deformations. Let

(i) $\Sigma$ be a set whose elements $\sigma$ are called states;

(ii) $\Pi$ be a set of functions $\pi:\left[0, d_{\pi}\right] \rightarrow \Sigma$, defined on the real interval $\left[0, d_{\pi}\right]$ (with $d_{\pi}>0$ ) that take their values in the state set; each element $\pi \in \Pi$ is called a process and the interval $\left[0, d_{\pi}\right]$ is interpreted as the time-interval during which the process takes place; $d_{\pi}$ is called the duration of the process; 
$\pi^{i}:=\pi(0)$ and $\pi^{f}:=\pi\left(d_{\pi}\right)$ denote the initial and the final values of $\pi$, respectively;

(iii) $\widehat{E}: \Sigma \rightarrow$ Sym be a mapping defined on the state set that takes its values in Sym, the space of the second-order symmetric tensors; for each state $\sigma$, $\widehat{E}(\sigma)$ is interpreted as the corresponding infinitesimal strain tensor, at a fixed material point, with respect to a fixed reference configuration;

(iv) $\widehat{S}: \Sigma \rightarrow$ Sym be a mapping that, at each state $\sigma$, delivers the corresponding stress $\widehat{S}(\sigma)$.

If the process set $\Pi$ satisfies the following two properties P1 and P2, the quadruple $\mathscr{M}=(\Sigma, \Pi, \widehat{E}, \widehat{S})$ is called a material element.

P1. If processes $\pi_{1}$ and $\pi_{2}$ are such that $\pi_{1}^{f}=\pi_{2}^{i}$, then $\Pi$ also contains process

$$
\pi_{1} * \pi_{2}(\tau):= \begin{cases}\pi_{1}(\tau), & \tau \in\left[0, d_{\pi_{1}}\right], \\ \pi_{2}\left(\tau-d_{\pi_{1}}\right), & \tau \in\left[d_{\pi_{1}}, d_{\pi_{1}}+d_{\pi_{2}}\right]\end{cases}
$$

of duration $\left(d_{\pi_{1}}+d_{\pi_{2}}\right) ; \pi_{1} * \pi_{2}$ is called the composition of $\pi_{1}$ with $\pi_{2}$.

P2. For each $\pi \in \Pi$ and for every couple $\tau_{1}, \tau_{2}$ with $0 \leq \tau_{1}<\tau_{2} \leq d_{\pi}$, $\Pi$ contains process

$$
\pi_{\left[\tau_{1}, \tau_{2}\right]}(\tau):=\pi\left(\tau+\tau_{1}\right), \quad \tau \in\left[0, \tau_{2}-\tau_{1}\right],
$$

of duration $\left(\tau_{2}-\tau_{1}\right) ; \pi_{\left[\tau_{1}, \tau_{2}\right]}$ is called the $\left[\tau_{1}, \tau_{2}\right]$-segment of $\pi$.

In view of properties (iii) and (iv) of the material element, each process $\pi$ determines the evolution of the strain and the stress, by means of mappings $\tau \mapsto \widehat{E}_{\pi}$ and $\tau \mapsto \widehat{S}_{\pi}$, defined on the interval $\left[0, d_{\pi}\right]$, which take their values in Sym,

$$
\widehat{E}_{\pi}:=\widehat{E} \circ \pi ; \quad \widehat{S}_{\pi}:=\widehat{S} \circ \pi .
$$

$\widehat{E}_{\pi}$ and $\widehat{S}_{\pi}$ are called, respectively, strain path and stress path corresponding to process $\pi$. Here we make the assumption that, for each $\pi \in \Pi, \widehat{E}_{\pi}$ and $\widehat{S}_{\pi}$ are Lipschitz continuous functions on $\left[0, d_{\pi}\right]$, with a right derivative at each $\tau \in\left[0, d_{\pi}[\right.$. We shall use $\dot{E}_{\pi}$ (or $\dot{S}_{\pi}$ ) to indicate the value of the time-derivative of $\widehat{E}_{\pi}$ (or $\widehat{S}_{\pi}$ ). For the value of $\tau$ for which the derivative of $\widehat{E}_{\pi}$ (or $\widehat{S}_{\pi}$ ) is not defined, we take $\dot{E}_{\pi}\left(\right.$ or $\left.\dot{S}_{\pi}\right)$ to denote the right derivative.

Let

$$
\mathfrak{F}(\sigma):=\left\{\left(\widehat{E}_{\pi}, \widehat{S}_{\pi}\right) \mid \pi \in \Pi, \pi^{i}=\sigma\right\}
$$

be the set of all the couples of strain and stress paths corresponding to the processes that begin in $\sigma$. Let us now proceed to state two important properties that a material element $\mathscr{M}=(\Sigma, \Pi, \widehat{E}, \widehat{S})$ may possess. $\Sigma$,

$\mathscr{M}$ is said to satisfy the minimality condition [8] if, for $\sigma_{1}$ and $\sigma_{2}$ belonging to

$$
\mathfrak{F}\left(\sigma_{1}\right)=\mathfrak{F}\left(\sigma_{2}\right) \quad \Rightarrow \quad \sigma_{1}=\sigma_{2} .
$$

The minimality condition is the assertion that from two distinct states at least two distinct couples of strain and stress paths originate; in other words, there are no irrelevant state variables. 
will be said to satisfy the unique lifting property [8] if any two processes $\pi_{1}$ and $\pi_{2}$, which have the same duration, begin from the same state, and determine the same strain path, coincide, i.e.,

$$
d_{\pi_{1}}=d_{\pi_{2}} ; \quad \pi_{1}^{i}=\pi_{2}^{i} ; \quad \widehat{E}_{\pi_{1}}=\widehat{E}_{\pi_{2}} \Rightarrow \pi_{1}=\pi_{2} .
$$

If the material element satisfies the unique lifting property, each process is entirely determined by the corresponding strain path.

2.2. Free-energy functions. This subsection deals with certain well-known results of thermodynamics. It should be remembered that the processes are assumed to be isothermal.

The material element $\mathscr{M}=(\Sigma, \Pi, \widehat{E}, \widehat{S})$ is said to have a base state $\sigma_{0}$ if $\sigma_{0} \in \Sigma$ exists, such that, for every other state $\sigma \in \Sigma$, there exists at least one process $\pi \in \Pi$ that begins in $\sigma_{0}$ and ends in $\sigma$, i.e., such that

$$
\pi^{i}=\sigma_{0} ; \quad \pi^{f}=\sigma
$$

(cf. [7]).

The material element $\mathscr{M}$ is said to satisfy the condition of perfect accessibility if each state is a base state, i.e., if, however two states $\sigma_{1}$ and $\sigma_{2}$ in $\Sigma$ are chosen, there exists at least one process $\pi \in \Pi$ such that we get

$$
\pi^{i}=\sigma_{1} ; \quad \pi^{f}=\sigma_{2} .
$$

For each $\pi \in \Pi$,

$$
w(\pi):=\int_{0}^{d_{\pi}} \widehat{S}_{\pi}(\tau) \cdot \dot{E}_{\pi}(\tau) d \tau
$$

is the work (per unit volume in the reference configuration) done by the exterior on the material element during process $\pi$. In relation $(2.7), \widehat{E}_{\pi}$ and $\widehat{S}_{\pi}$ are, respectively, the strain and stress paths corresponding to $\pi$, defined by relation (2.1); moreover, for $A$ and $B$ belonging to $\operatorname{Sym}, A \cdot B:=\operatorname{tr}(A B)$, with $\operatorname{tr}$ indicating the trace, denotes the inner product between $A$ and $B$.

A material element with a base state $\sigma_{0}$ is said to satisfy the second law of thermodynamics [7] if, for each state $\sigma \in \Sigma$, there exists a real constant $\kappa(\sigma)$ with the property that

$$
w(\pi) \geq \kappa(\sigma), \text { for each } \pi \in \Pi, \text { such that } \pi^{i}=\sigma_{0} \text { and } \pi^{f}=\sigma .
$$

Let $\psi: \Sigma \rightarrow \mathbb{R}$ be a real estate function; $\psi$ is said to be a free-energy function of the material element if

$$
w(\pi) \geq \psi\left(\pi^{f}\right)-\psi\left(\pi^{i}\right) \text { for every } \pi \in \Pi .
$$

The inequality (2.9) is called the internal dissipation inequality. It is well known that certain material elements may be endowed with several free-energy functions which do not differ by a constant alone. The material element to be studied below demonstrates this abundantly.

A function $\psi$ is said to be regular if, for each process $\pi$, function $\psi_{\pi}:=\psi \circ$ $\pi:\left[0, d_{\pi}\right] \rightarrow \mathbb{R}$ is Lipschitz continuous on $\left[0, d_{\pi}\right]$ and has a right derivative for each 
$\tau \in\left[0, d_{\pi}\right.$ [. If $\psi$ is regular and satisfies (2.9), in view of P2 and of the regularity stipulated for $\widehat{S}_{\pi}$ and $\widehat{E}_{\pi}$, for every process $\pi$ we have

$$
\widehat{S}_{\pi}(\tau) \cdot \dot{E}_{\pi}(\tau)-\dot{\psi}_{\pi}(\tau) \geq 0 \text { for each } \tau \in\left[0, d_{\pi}[,\right.
$$

which constitutes the local form of the internal dissipation inequality. The fundamental property of the free-energy functions is stated by means of the following proposition [7].

Proposition 2.1. A material element with a base state satisfies the second law of thermodynamics in the formulation (2.8) if, and only if, it has at least one freeenergy function.

When proving Proposition 2.1, a free-energy function is constructed; to be precise, on the assumption that the element has $\sigma_{0}$ as its base state and that it satisfies the second law, it is shown that the real function

$$
\underline{\psi}(\sigma):=\inf \left\{w(\pi) \mid \pi \in \Pi ; \pi^{i}=\sigma_{0}, \pi^{f}=\sigma\right\}, \quad \underline{\psi}\left(\sigma_{0}\right)=0
$$

is well defined on $\Sigma$ and satisfies the dissipation inequality (2.9) for every process $\pi$. Moreover, as the following proposition states, $\underline{\psi}$ is an upper bound for the set of all free-energy functions vanishing at $\sigma_{0}$.

Proposition 2.2. Let $\sigma_{0}$ be a base state for a material element and let $\underline{\psi}$ be the free-energy function defined by (2.11). Then, for any free-energy function $\psi$ with $\psi\left(\sigma_{0}\right)=0$, we have

$$
\psi \leq \underline{\psi} .
$$

For a material element that satisfies the condition of perfect accessibility, it is possible to give a formulation of the second law of thermodynamics that is equivalent to (2.8) in every way, but, from certain points of view, more convenient. This result is established by means of the next Proposition 2.3 [6].

A process $\pi$ is said to be cyclic if the initial state of the process coincides with the final state, i.e., if

$$
\pi^{i}=\pi^{f}
$$

It should be noted that, if the material element satisfies the condition of perfect accessibility, in view of $\mathrm{P} 2$, for each process $\pi$ there exists a cyclic process $\tilde{\pi}$ such that $\pi$ is a segment of $\tilde{\pi}$.

Proposition 2.3. A material element with perfect accessibility satisfies the second law of thermodynamics, in formulation (2.8), if and only if, for each cyclic process $\pi$, we have

$$
w(\pi) \geq 0 .
$$

Of course, for each state $\sigma_{0}$ of a material element with perfect accessibility, function $\psi$ defined by $(2.11)$ is a free-energy function for the material element. With the following proposition, we establish that, for a material element with perfect accessibility, it is possible to determine a lower bound and an upper bound for all the free-energy functions that vanish at the same state [6]. 
Proposition 2.4. Let $\sigma_{0}$ be any state of a material element with perfect accessibility that satisfies the second law of thermodynamics. Then, the function $\bar{\psi}: \Sigma \rightarrow \mathbb{R}$ defined by

$$
\bar{\psi}(\sigma):=\sup \left\{-w(\pi) \mid \pi \in \Pi ; \pi^{i}=\sigma, \pi^{f}=\sigma_{0}\right\}, \quad \bar{\psi}\left(\sigma_{0}\right)=0
$$

is a free-energy function for the material element.

Moreover, if $\psi: \Sigma \rightarrow \mathbb{R}$ is any other free-energy function, such that $\psi\left(\sigma_{0}\right)=0$, we have

$$
\underline{\psi}(\sigma) \geq \psi(\sigma) \geq \bar{\psi}(\sigma) \text { for each } \sigma \in \Sigma .
$$

3. Von Mises elastic-plastic materials. To start with, this section will consist in an examination of the properties of the isotropic elastic-plastic element, with combined work-hardening, that satisfies the von Mises yield criterion. This will be followed by an analysis of the case of a material that hardens kinematically, following the classic Melan rule. We shall confine ourselves to the case of infinitesimal isothermal deformations; for a more general discussion of this and addition references, see [9$11]$.

3.1. Combined work-hardening. For each $A \in \mathrm{Sym}$,

$$
A_{0}:=A-\frac{1}{3}(\operatorname{tr} A) I
$$

denotes the deviator of $A$. Similarly,

$$
\mathrm{Sym}_{0}:=\{A \in \operatorname{Sym} \mid \operatorname{tr} A=0\}
$$

denotes the set of all the traceless symmetric tensors.

Let $\tilde{\rho}: \mathbb{R}^{+} \rightarrow \mathbb{R}^{+}$be a strictly increasing, differentiable function such that $\tilde{\rho}(0)>0$ and let $\eta \geq 0$. Let us consider a material element whose set of states

$$
\Sigma:=\left\{\left(E, E^{p}, \zeta\right) \in \operatorname{Sym} \times \operatorname{Sym}_{0} \times \mathbb{R}^{+} \mid \zeta \geq\left\|E^{p}\right\| \text { and }\left\|E_{0}-(1+\eta) E^{p}\right\| \leq \tilde{\rho}(\zeta)\right\},
$$

is made up of all the triples $\left(E, E^{p}, \zeta\right)$, where $E$ and $E^{p}$ belong to Sym and $\mathrm{Sym}_{0}$, respectively, and the distance between the deviator $E_{0}$ of $E$ and $(1+\eta) E^{p}$ is not greater than $\tilde{\rho}(\zeta)$; $\zeta$ is called the Odqvist parameter and is a number not smaller than $\left\|E^{p}\right\|$.

$E$ and $E^{p}$ are interpreted as the infinitesimal strain and the plastic strain [1] corresponding to $\sigma$, respectively, so that, in view of item (iii) of the definition of the material element, we have

$$
\widehat{E}(\sigma)=E .
$$

By the hypothesis that $E^{p}$ belongs to $\mathrm{Sym}_{0}$ we assume there is no plastic change in volume.

Let us use $\widehat{E}^{p}$ and $\hat{\zeta}$ to denote the mappings that assign to each state $\sigma \in \Sigma$ the corresponding plastic strain and the corresponding Odqvist parameter, respectively.

$$
\widehat{E}^{p}: \Sigma \rightarrow \operatorname{Sym}_{0}, \quad E^{p}=\widehat{E}^{p}(\sigma) ; \quad \hat{\zeta}: \Sigma \rightarrow \mathbb{R}^{+}, \quad \zeta=\hat{\zeta}(\sigma) .
$$

The hypothesis is put forward here that the stress $\widehat{S}(\sigma)$ corresponding to state $\sigma$ depends solely on the value of the corresponding strain and plastic strain, a hypothesis 
that reflects common experience with many materials of interest in applications, in that the plastic deformations do not change the elastic moduli. Moreover, since we are dealing with isotropic materials and infinitesimal deformations, we suppose two material constants $\lambda$ and $\mu$ exist, with $\mu>0,2 \mu+3 \lambda>0$, such that, for each $\sigma=\left(E, E^{p}, \zeta\right)$, we have

$$
\widehat{S}(\sigma)=\mathbb{S}\left[\widehat{E}(\sigma)-\widehat{E}^{p}(\sigma)\right]:=2 \mu\left(\widehat{E}(\sigma)-\widehat{E}^{p}(\sigma)\right)+(\lambda \operatorname{tr} \widehat{E}(\sigma)) I .
$$

As in the elastic case, $\lambda$ and $\mu$ are called Lamé moduli.

The set $\Pi$ of the processes is made up of mappings $\pi:\left[0, d_{\pi}\right] \rightarrow \Sigma$ such that, given

$$
\begin{aligned}
\widehat{E}_{\pi}^{p} & :=\widehat{E}^{p} \circ \pi, \\
\hat{\zeta}_{\pi} & :=\hat{\zeta} \circ \pi, \\
\widehat{C}_{\pi} & :=(1+\eta) \widehat{E}_{\pi}^{p}, \\
\widehat{N}_{\pi} & :=\left(1 / \tilde{\rho}\left(\zeta_{\pi}\right)\right)\left(\left(\widehat{E}_{\pi}\right)_{0}-\widehat{C}_{\pi}\right),
\end{aligned}
$$

and using $\tilde{\rho}^{\prime}$ to denote the derivative of $\tilde{\rho}$, the system [1]

$$
\begin{aligned}
& \dot{E}_{\pi}^{p}(\tau)=\dot{\zeta}_{\pi}(\tau) \widehat{N}_{\pi}(\tau),
\end{aligned}
$$

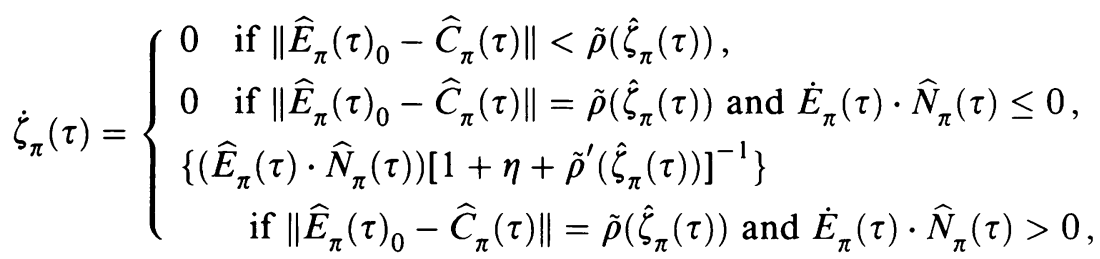

with the initial conditions

$$
\widehat{E}_{\pi}^{p}(0)=\widehat{E}^{p}\left(\pi^{i}\right), \quad \hat{\zeta}_{\pi}(0)=\hat{\zeta}\left(\pi^{i}\right)
$$

has one and only one Lipschitz continuous solution on $\left[0, d_{\pi}\right]$, with a right derivative for each $\tau \in\left[0, d_{\pi}\left[{ }^{1}\right.\right.$

From (3.7)-(3.13) it is easy to deduce that the set $\Pi$ of the processes so defined satisfies conditions P1 and P2. Moreover, with Proposition 3.1 below, it will be proved that $\Pi$ is not empty. Therefore, the quadruple $(\Sigma, \Pi, \widehat{E}, \widehat{S})$, with $\widehat{E}$ and $\widehat{S}$ defined by (3.4) and (3.6), respectively, constitutes a material element which will be denoted by $\mathscr{M}_{c}$.

We observe that each process $\pi$ can be identified with the triple of the corresponding mappings $\left(\widehat{E}_{\pi}, \widehat{E}_{\pi}^{p}, \hat{\zeta}_{\pi}\right)$ and, in view of the uniqueness of the solution of system (3.11)-(3.13), is univocally determined by $\widehat{E}_{\pi}$. Therefore (2.4) proves to be verified and $\mathscr{M}_{c}$ satisfies the unique lifting property.

For each $\sigma=\left(E, E^{p}, \zeta\right) \in \Sigma$, the cylinder

$$
E(\sigma):=\left\{A \in \operatorname{Sym} \mid\left\|A_{0}-(1+\eta) E^{p}\right\| \leq \tilde{\rho}(\zeta)\right\}
$$

\footnotetext{
${ }^{1}$ Unlike materials with kinematic work-hardening (see the following Proposition 3.3), I know of no existence and uniqueness theorem for system (3.11)-(3.13) sufficiently general to include the cases most often encountered in applications.
} 
with its center $C:=(1+\eta) E^{p}$ and radius $\tilde{\rho}(\zeta)$ is the elastic range corresponding to $\sigma$ (cf. [3] and [11]). In view of (3.3), $E(\sigma)$ contains $\widehat{E}(\sigma)$, while it is not required to contain $\widehat{E}^{p}(\sigma) . E(\sigma)$ depends on $\widehat{E}^{p}(\sigma)$ and $\hat{\zeta}(\sigma)$ alone and is constituted by the symmetric gradients of all deformations from the reference configuration to configurations that are elastically accessible from the current configuration. Indeed, if we confine our attention to processes $\pi$ whose corresponding strain path $\widehat{E}_{\pi}$ remains within the elastic range $E\left(\pi^{i}\right)$ corresponding to $\pi^{i}$, i.e., such that

$$
\widehat{E}_{\pi}(\tau) \in E\left(\pi^{i}\right) \text { for each } \tau \in\left[0, d_{\pi}\right],
$$

it can be deduced from (3.11) and (3.12) that

$$
\dot{\zeta}_{\pi}(\tau)=0 ; \quad \dot{E}_{\pi}^{p}(\tau)=0 \text { for each } \tau \in\left[0, d_{\pi}[.\right.
$$

It therefore follows from (3.6) that

$$
\widehat{S}_{\pi}(\tau)=2 \mu\left(\widehat{E}_{\pi}(\tau)-\widehat{E}^{p}\left(\pi^{i}\right)\right)+\left(\lambda \operatorname{tr} \widehat{E}_{\pi}(\tau)\right) I, \quad \tau \in\left[0, d_{\pi}\right],
$$

and from (3.14) that

$$
E(\pi(\tau))=E\left(\pi^{i}\right), \quad \tau \in\left[0, d_{\pi}\right] .
$$

Every process $\pi$ that satisfies (3.15) is called an elastic process.

Relation (3.11) constitutes the classic associated flow rule according to which $\dot{E}^{p}(\tau)$ is either null or is parallel to $\widehat{N}_{\pi}(\tau)$, the outward unit normal field on elastic range $E(\pi(\tau))$ at $\widehat{E}_{\pi}(\tau)$. Then, from (3.11), it can be deduced that

$$
\hat{\zeta}_{\pi}\left(d_{\pi}\right)-\hat{\zeta}_{\pi}(0)=\int_{0}^{d_{\pi}}\left\|\dot{E}_{\pi}^{p}(\tau)\right\| d \tau
$$

is the length of the plastic strain path $\widehat{E}_{\pi}^{p}$ corresponding to $\pi$.

From (3.9) and (3.14), respectively, we obtain the isotropic work-hardening rule

$$
\dot{\tilde{\rho}}\left(\zeta_{\pi}\right)=\tilde{\rho}^{\prime}\left(\zeta_{\pi}\right) \dot{\zeta}_{\pi}
$$

and the kinematic work-hardening rule, similar to the one proposed by Melan [12],

$$
\dot{C}_{\pi}=(1+\eta) \dot{E}_{\pi}^{p} .
$$

In view of (3.14), (3.20), and (3.21) we shall call $\mathscr{M}_{c}$ a von Mises elastic-plastic material element with combined work-hardening. In particular, if we have $\eta=0$ we obtain a material element with isotropic work-hardening [13]; in this special case, it follows from (3.14) that, for each state $\sigma \in \Sigma$, the center of the elastic range coincides with plastic strain $E^{p}$.

The result proved by means of the following proposition is crucial for study of the thermodynamic properties of the material element.

Proposition 3.1. Let $\sigma_{1}=\left(E_{1}, E_{1}^{p}, \zeta_{1}\right)$ and $\sigma_{2}=\left(E_{2}, E_{2}^{p}, \zeta_{2}\right)$ be two states of the material element $\mathscr{M}_{c}$. A process $\pi$ such that $\pi^{i}=\sigma_{1}$ and $\pi^{f}=\sigma_{2}$ exists if and only if

$$
\zeta_{2}-\zeta_{1} \geq\left\|E_{2}^{p}-E_{1}^{p}\right\| .
$$


Proof. Necessity is an immediate consequence of (3.19). We shall prove sufficiency in four steps; let us begin with the case in which we have

$$
E_{2} \in E\left(\sigma_{1}\right)
$$

and therefore, in view of (3.16), $E_{2}^{p}=E_{1}^{p} ; \zeta_{2}=\zeta_{1}$. In this case, it is possible to construct an elastic process $\pi$ that starts in $\sigma_{1}$ and ends in $\sigma_{2}$. Indeed, for this purpose, it is enough to put $\widehat{E}_{\pi}(\tau)=\tau E_{2}+(1-\tau) E_{1}, \tau \in[0,1]$, and from (3.12) we can immediately deduce

$$
\widehat{E}_{\pi}^{p}(\tau)=E_{1}^{p} ; \quad \hat{\zeta}_{\pi}(\tau)=\zeta_{1}, \quad \tau \in[0,1],
$$

so that $\pi:=\left(\widehat{E}_{\pi}, E_{1}^{p}, \zeta_{1}\right)$ is the required process. Let us now prove sufficiency in the case in which $\zeta_{2}-\zeta_{1}=\left\|E_{2}^{p}-E_{1}^{p}\right\|$.

Given that $\alpha:=\left\|E_{2}^{p}-E_{1}^{p}\right\|, M:=\left(E_{2}^{p}-E_{1}^{p}\right) / \alpha$, let $A_{1}$ and $A_{2}$ be the elements of $\mathrm{Sym}_{0}$ defined as follows:

$$
A_{1}:=(1+\eta) E_{1}^{p}+\tilde{\rho}\left(\zeta_{1}\right) M ; \quad A_{2}:=(1+\eta) E_{2}^{p}+\tilde{\rho}\left(\zeta_{2}\right) M .
$$

We will prove that the required process can be obtained from the composition of three processes $\pi_{1}, \pi_{2}$, and $\pi_{3}$, where:

$\pi_{1}$ is any elastic process such that $\pi_{1}^{i}=\sigma_{1}, \pi_{1}^{f}=\bar{\sigma}_{1}:=\left(A_{1}, E_{1}^{p}, \zeta_{1}\right)$;

$\pi_{2}$ is a process such that $\pi_{2}^{i}=\bar{\sigma}_{1}, \pi_{2}^{f}=\bar{\sigma}_{2}:=\left(A_{2}, E_{2}^{p}, \zeta_{2}\right)$;

$\pi_{3}$ is any elastic process such that $\pi_{3}^{i}=\bar{\sigma}_{2}, \pi_{3}^{f}=\sigma_{2}$.

In view of (3.14) and (3.23), $A_{1} \in E\left(\sigma_{1}\right), A_{2} \in E\left(\sigma_{2}\right)$ and therefore the elastic processes $\pi_{1}$ and $\pi_{3}$ exist, as has been shown in the first step of the proof. The existence of $\pi_{2}$ remains, then, to be proved.

For this purpose, let us consider the mapping $\widetilde{E}:[0,1] \rightarrow \operatorname{Sym}_{0}$,

$$
\widetilde{E}(\tau):=(1+\eta)\left(\tau E_{2}^{p}+(1-\tau) E_{1}^{p}\right)+\tilde{\rho}\left(\zeta_{1}+\alpha \tau\right) M,
$$

which satisfies the relations

$$
\widetilde{E}(0)=A_{1} ; \quad \widetilde{E}(1)=A_{2} ; \quad \dot{\tilde{E}}(\tau)=\alpha\left[(\dot{1}+\eta)+\tilde{\rho}^{\prime}\left(\zeta_{1}+\alpha \tau\right)\right] M .
$$

Direct calculation proves that, given $\widehat{E}_{\pi_{2}}:=\widetilde{E}$, the functions

$$
\hat{E}_{\pi_{2}}^{p}(\tau):=\tau E_{2}^{p}+(1-\tau) E_{1}^{p}, \quad \hat{\zeta}_{\pi_{2}}(\tau)=\zeta_{1}+\alpha \tau, \quad \tau \in[0,1[,
$$

satisfy equations (3.11) and (3.12), respectively, with the initial conditions $\widehat{E}_{\pi_{2}}^{p}(0)=$ $E_{1}^{p}, \hat{\zeta}_{\pi_{2}}(0)=\zeta_{1}$. Given $\pi_{2}:=\left(\widehat{E}_{\pi_{2}}, \widehat{E}_{\pi_{2}}^{p}, \hat{\zeta}_{\pi_{2}}\right)$,

$$
\pi:=\pi_{1} * \pi_{2} * \pi_{3}
$$

the existence of which is guaranteed by property $\mathrm{P} 1$, is the required process.

Let us now prove sufficiency in the case in which $\sigma_{1}$ and $\sigma_{2}$ have the same corresponding plastic strain, i.e.,

$$
\sigma_{1}=\left(E_{1}, E^{p}, \zeta_{1}\right), \quad \sigma_{2}=\left(E_{2}, E^{p}, \zeta_{2}\right), \quad \text { with } \zeta_{2}>\zeta_{1} .
$$


Given that $N:=E^{p} /\left\|E^{p}\right\|$ (if $E^{p}=0, N$ can be arbitrarily chosen among the traceless unit tensors), for $\alpha>0$, we put

$$
\begin{gathered}
\widetilde{E}_{1}:[0,1] \rightarrow \operatorname{Sym}_{0}, \quad \widetilde{E}_{1}(\tau):=(1+\eta)\left(E^{p}+\alpha \tau N\right)+\tilde{\rho}\left(\zeta_{1}+\alpha \tau\right) N, \\
\widetilde{E}_{2}:[0,1] \rightarrow \operatorname{Sym}_{0}, \quad \widetilde{E}_{2}(\tau):=(1+\eta)\left(E^{p}+\alpha(1-\tau) N\right)-\tilde{\rho}\left(\zeta_{1}+\alpha(1+\tau)\right) N .
\end{gathered}
$$

Given $\widehat{E}_{\pi_{1}}:=\widetilde{E}_{1}, \widehat{E}_{\pi_{3}}:=\widetilde{E}_{2}$, from (3.11) and (3.12) we obtain

$$
\begin{aligned}
\hat{E}_{\pi_{1}}^{p}(\tau)=E^{p}+\alpha \tau N, & \hat{\zeta}_{\pi_{1}}(\tau)=\zeta_{1}+\alpha \tau ; \\
\widehat{E}_{\pi_{3}}^{p}(\tau)=E^{p}+\alpha(1-\tau) N, & \hat{\zeta}_{\pi_{3}}(\tau)=\zeta_{1}+\alpha(1+\tau) .
\end{aligned}
$$

Thus, if we use $\pi_{2}$ to denote the elastic process that links states $\sigma_{3}=\left(\widehat{E}_{\pi_{1}}(1), E^{p}+\right.$ $\left.\alpha N, \zeta_{1}+\alpha\right)$ and $\sigma_{4}=\left(\widehat{E}_{\pi_{2}}(0), E^{p}+\alpha N, \zeta_{1}+\alpha\right)$ and if we put

$$
\alpha=\frac{1}{2}\left(\zeta_{2}-\zeta_{1}\right), \quad \bar{\pi}:=\pi_{1} * \pi_{2} * \pi_{3}
$$

we get

$$
\bar{\pi}^{i}=\sigma_{5}=:\left(\bar{E}_{1}, E^{p}, \zeta_{1}\right), \quad \bar{\pi}^{f}=\sigma_{6}=:\left(\bar{E}_{2}, E^{p}, \zeta_{2}\right)
$$

where

$$
\bar{E}_{1}=:(1+\eta) E^{p}+\tilde{\rho}\left(\zeta_{1}\right) N, \quad \bar{E}_{2}=:(1+\eta) E^{p}-\tilde{\rho}\left(\zeta_{2}\right) N .
$$

On the other hand, $E_{1}$ belongs to $E\left(\sigma_{5}\right)=E\left(\sigma_{1}\right)$ and therefore also to $E\left(\sigma_{6}\right)$, because $\tilde{\rho}\left(\zeta_{2}\right)>\tilde{\rho}\left(\zeta_{1}\right)$. We can thus construct two elastic processes $\pi_{4}$ and $\pi_{5}$ such that $\pi_{4}^{i}=\sigma_{1}, \pi_{4}^{f}=\sigma_{5} ; \pi_{5}^{i}=\sigma_{6}, \pi_{5}^{f}=\sigma_{2}$ and, therefore,

$$
\pi:=\pi_{4} * \bar{\pi} * \pi_{5}
$$

is the required process.

Lastly, let us consider the general case in which $\zeta_{2}-\zeta_{1}>\left\|E_{2}^{p}-E_{1}^{p}\right\|$. Let $\sigma^{*}:=$ $\left((1+\eta) E_{2}^{p}, E_{2}^{p}, \zeta_{1}+\left\|E_{2}^{p}-E_{1}^{p}\right\|\right)$. In view of the second and third steps of this proof, respectively, there exist two processes $\tilde{\pi}_{1}$ and $\tilde{\pi}_{2}$ such that $\tilde{\pi}_{1}^{i}=\sigma_{1}, \tilde{\pi}_{1}^{f}=\sigma^{*}$; $\tilde{\pi}_{2}^{i}=\sigma^{*}, \tilde{\pi}_{2}^{f}=\sigma_{2}$. Thus

$$
\pi:=\tilde{\pi}_{1} * \tilde{\pi}_{2}
$$

is the required process.

With the following proposition we shall prove that no irrelevant state variables are introduced in defining the material element $\mathscr{M}_{c}$.

Proposition 3.2. The material element $\mathscr{M}_{c}$ satisfies the minimality condition.

Proof. Let us suppose $\sigma_{1} \neq \sigma_{2}$. We wish to prove that $\mathfrak{F}\left(\sigma_{1}\right) \neq \mathfrak{F}\left(\sigma_{2}\right)$. In view of the definition of $\Pi$, it is sufficient to confine ourselves to considering states $\sigma_{1}=$ $\left(E, E^{p}, \zeta_{1}\right), \sigma_{2}=\left(E, E^{p}, \zeta_{2}\right)$, with $\zeta_{2}>\zeta_{1}$, which differ only as far as the value of the corresponding Odqvist parameter is concerned. We shall omit the details of the proof here, confining ourselves to observing that, in view of (3.14) and the fact that $\tilde{\rho}$ is a strictly increasing function ${ }^{2}, E\left(\sigma_{2}\right)$ and $E\left(\sigma_{1}\right)$ are two concentric cylinders,

${ }^{2}$ It should be noted that the fact that $\tilde{\rho}$ is a strictly increasing function is crucial for the proof of this proposition. 
the first of which contains the second. Therefore, any straight strain path starting in $E$, similar to those constructed during the proof of Proposition 3.1 above, intersects the boundary of $E\left(\sigma_{2}\right)$ after the boundary of $E\left(\sigma_{1}\right)$. In view of (3.11) and (3.12), this latter fact implies $\mathfrak{F}\left(\sigma_{1}\right) \neq \mathfrak{F}\left(\sigma_{2}\right)$.

3.2. Kinematic work-hardening. In this subsection we shall deal with an elasticplastic material for which the radius of the elastic range does not depend on the state. In these circumstances, therefore, the state is characterized entirely by the strain and plastic strain. Let

$$
\Sigma:=\left\{\sigma=\left(E, E^{p}\right) \in \operatorname{Sym} \times \operatorname{Sym}_{0} \mid\left\|E_{0}-(1+\eta) E^{p}\right\| \leq \rho_{0}\right\},
$$

where $\rho_{0}$ is a positive material constant, the set of the states.

The set $\Pi$ of the processes is made up of the couples of strain and plastic strain paths,

$$
\pi=\left(\widehat{E}_{\pi}, \widehat{E}_{\pi}^{p}\right):\left[0, d_{\pi}\right] \rightarrow \operatorname{Sym} \times \operatorname{Sym}_{0},
$$

such that $\widehat{E}_{\pi}$ is a Lipschitz continuous function on $\left[0, d_{\pi}\right]$, with derivative of bounded total variation and $\widehat{E}_{\pi}^{p}$ satisfies the following relations $(3.36)-(3.38)$ :

$$
\widehat{E}_{\pi}^{p}(0)=\widehat{E}^{p}\left(\pi^{i}\right)
$$

and, for $\tau \in\left[0, d_{\pi}[\right.$,

$$
\begin{gathered}
\dot{E}_{\pi}^{p}(\tau)=\left\|\dot{E}_{\pi}^{p}(\tau)\right\| \widehat{N}_{\pi}(\tau), \\
\left\|\dot{E}_{\pi}^{p}(\tau)\right\|=\left\{\begin{array}{rr}
0 & \text { if }\left\|\widehat{E}_{\pi}(\tau)_{0}-\widehat{C}_{\pi}(\tau)\right\|<\rho_{0}, \\
0 & \text { if }\left\|\widehat{E}_{\pi}(\tau)_{0}-\widehat{C}_{\pi}(\tau)\right\|=\rho_{0} \text { and } \dot{E}_{\pi}(\tau) \cdot \widehat{N}_{\pi}(\tau) \leq 0, \\
\left\{\left(\widehat{E}_{\pi}(\tau) \cdot \widehat{N}_{\pi}(\tau)\right)[1+\eta]^{-1}\right\} & \text { if }\left\|\widehat{E}_{\pi}(\tau)_{0}-\widehat{C}_{\pi}(\tau)\right\|=\rho_{0}
\end{array}\right. \\
\text { and } \dot{E}_{\pi}(\tau) \cdot \widehat{N}_{\pi}(\tau)>0,
\end{gathered}
$$

where, as in the case of combined work-hardening materials, we put

$$
\widehat{C}_{\pi}:=(1+\eta) \widehat{E}_{\pi}^{p} ; \quad \widehat{N}_{\pi}:=\left(1 / \rho_{0}\right)\left(\left(\widehat{E}_{\pi}\right)_{0}-\widehat{C}_{\pi}\right) .
$$

In this case, in the definition of $\Pi$ it has not been necessary to require the existence, uniqueness, and regularity of the solution of the system (3.36)-(3.38) because these are ensured by the following proposition, the proof of which is similar to that of Proposition (3.3) in [14] and, consequently, has been omitted.

Proposition 3.3. Let us suppose $\widehat{E}_{\pi}$ is a Lipschitz continuous function on $\left[0, d_{\pi}\right]$, with the derivative of bounded variation. The system (3.37), (3.38), with initial condition (3.36), has a unique Lipschitz continuous solution $\widehat{E}_{\pi}^{p}$ on $\left[0, d_{\pi}\right]$, with a right derivative for each $\tau \in\left[0, d_{\pi}[\right.$.

For each state $\sigma$, the corresponding elastic range is the cylinder

$$
E(\sigma)=\left\{A \in \operatorname{Sym} \mid\left\|A_{0}-(1+\eta) E_{p}\right\| \leq \rho_{0}\right\}
$$

whose center $C=(1+\eta) E^{p}$ follows evolution law (3.21).

Such a material element is called a von Mises elastic-plastic material element with kinematic work-hardening and is indicated by $\mathscr{M}_{k}$. In particular, if we have $\eta=0$, the material is said to be ideally plastic. 
The next proposition follows directly from the definition of $\Pi$.

Proposition 3.4. The material element $\mathscr{M}_{k}$ satisfies the minimality condition and unique lifting property.

Unlike what happens in the case of materials with isotropic work-hardening, the radius of the elastic range is a material constant and, therefore, the Odqvist parameter $\zeta$ is not needed to define the state of the material element. If, in this case too, we had included $\zeta$ among the state variables, the element thus constructed would not have satisfied the minimality condition. Of course, $\mathscr{M}_{k}$ cannot be obtained as a special case of $\mathscr{M}_{c}$ by choosing $\tilde{\rho}^{\prime}=0$.

4. Thermodynamic properties of material elements $\mathscr{M}_{c}$ and $\mathscr{M}_{k}$. In this section, we prove that elements $\mathscr{M}_{c}$ and $\mathscr{M}_{k}$ satisfy the second law of thermodynamics and we determine the corresponding free-energy functions.

4.1. The material element $\mathscr{M}_{c}$. As proved by Proposition 3.1, $\Pi$ does not contain processes that begin at a state to which an Odqvist parameter corresponds that is greater than the one corresponding to the final state, and therefore $\mathscr{M}_{c}$ is not a material element with perfect accessibility. In spite of this, $\mathscr{M}_{c}$ has a base state, as is proved by the next proposition.

Let $\sigma_{0}$ be the state to which null strain, plastic strain and the Odqvist parameter correspond, i.e., $\sigma_{0}:=(0,0,0) ; \sigma_{0}$ is called the annealed state of the material element $\mathscr{M}_{c}$. A free-energy function $\psi$ for $\mathscr{M}_{c}$ is said to be normalized if $\psi\left(\sigma_{0}\right)=$ 0 .

Proposition 4.1. The annealed state is a base state for the material element $\mathscr{M}_{c}$.

Proof. The desired result follows directly from Proposition 3.1, if we observe that, in view of (3.3), for each state $\sigma=\left(E, E^{p}, \zeta\right)$ we have $\zeta \geq\left\|E^{p}\right\|$.

Proposition 4.2. Let $\sigma_{1}=\left(E_{1}, E_{1}^{p}, \zeta_{1}\right)$ and $\sigma_{2}=\left(E_{2}, E_{2}^{p}, \zeta_{2}\right)$ be two states and let $\pi$ be a process such that $\pi^{i}=\sigma_{1}$ and $\pi^{f}=\sigma_{2}$. Then, we have

$$
\begin{aligned}
w(\pi)= & \frac{1}{2}\left\{\left(E_{2}-E_{2}^{p}\right) \cdot \mathbb{S}\left[E_{2}-E_{2}^{p}\right]-\left(E_{1}-E_{1}^{p}\right) \cdot \mathbb{S}\left[E_{1}-E_{1}^{p}\right]\right\} \\
& +\mu \eta\left\{\left\|E_{2}^{p}\right\|^{2}-\left\|E_{1}^{p}\right\|^{2}\right\}+2 \mu\left\{\tilde{\omega}\left(\zeta_{2}\right)-\tilde{\omega}\left(\zeta_{1}\right)\right\}
\end{aligned}
$$

where $\tilde{\omega}$ is a primitive of $\tilde{\rho}$.

Proof. From (2.1), (2.7), (3.6), and (3.7) we deduce

$$
\begin{aligned}
w(\pi) & =\int_{0}^{d_{\pi}} \widehat{S}_{\pi}(\tau) \cdot \dot{E}_{\pi}(\tau) d \tau \\
& =\int_{0}^{d_{\pi}} \dot{E}_{\pi}(\tau) \cdot \mathbb{S}\left[\widehat{E}_{\pi}(\tau)-\widehat{E}_{\pi}^{p}(\tau)\right] d \tau \\
& =\int_{0}^{d_{\pi}}\left\{\left(\dot{E}_{\pi}(\tau)-\dot{E}_{\pi}^{p}(\tau)\right) \cdot \mathbb{S}\left[\widehat{E}_{\pi}(\tau)-\widehat{E}_{\pi}^{p}(\tau)\right]+\dot{E}_{\pi}^{p}(\tau) \cdot \mathbb{S}\left[\widehat{E}_{\pi}(\tau)-\widehat{E}_{\pi}^{p}(\tau)\right]\right\} d \tau \\
& =\left[\left(E-E^{p}\right) \cdot \mathbb{S}\left[E-E^{p}\right]\right]_{0}^{d_{\pi}} / 2+\int_{0}^{d_{\pi}} 2 \mu\left(\widehat{E}_{\pi}(\tau)-\widehat{E}_{\pi}^{p}(\tau)\right) \cdot \dot{E}_{\pi}^{p}(\tau) d \tau .
\end{aligned}
$$


Moreover, from (3.8)-(3.13) we obtain

$$
\begin{aligned}
\int_{0}^{d_{\pi}} & 2 \mu\left(\widehat{E}_{\pi}(\tau)-\widehat{E}_{\pi}^{p}(\tau)\right) \cdot \dot{E}_{\pi}^{p}(\tau) d \tau \\
& =\int_{0}^{d_{\pi}} 2 \mu \tilde{\rho}\left(\zeta_{\pi}(\tau)\right) \widehat{N}_{\pi}(\tau) \cdot \dot{E}_{\pi}^{p}(\tau) d \tau+\int_{0}^{d_{\pi}} 2 \mu \eta \widehat{E}_{\pi}^{p}(\tau) \cdot \dot{E}_{\pi}^{p}(\tau) d \tau \\
& =\left[2 \mu \tilde{\omega}(\zeta)+\mu \eta\left\|\widehat{E}^{p}\right\|^{2}\right]_{0}^{d_{\pi}} \cdot \quad
\end{aligned}
$$

Relation (4.1) shows that for every process $\pi$, the work $w(\pi)$ depends on $\pi^{i}$ and $\pi^{f}$ alone. Consequently, in view of (2.11), we have

$$
\underline{\psi}(\sigma)=\left(E-E^{p}\right) \cdot \mathbb{S}\left[E-E^{p}\right] / 2+\mu \eta\left\|E^{p}\right\|^{2}+2 \mu \tilde{\omega}(\zeta),
$$

where $\hat{\omega}$ is the primitive of $\tilde{\rho}$ such that $\hat{\omega}(0)=0$. Therefore $\psi$ is a normalized free-energy function for $\mathscr{M}_{c}$ with the special property of satisfying relation (2.9) with an equality. In particular, for a material element with isotropic work-hardening we have

$$
\underline{\psi}(\sigma)=\left(E-E^{p}\right) \cdot \mathbb{S}\left[E-E^{p}\right] / 2+2 \mu \hat{\omega}(\zeta) .
$$

The following proposition is an immediate consequence of Proposition 2.1 and of relations (2.9), (4.1), and (4.2).

Proposition 4.3. The element $\mathscr{M}_{c}$ satisfies the second law of thermodynamics. Any normalized free-energy function $\psi$ corresponding to $\mathscr{M}_{c}$ satisfies $\psi \leq \underline{\psi}$.

The following Propositions 4.4 and 4.5 supply the required characterization of the free-energy functions corresponding to the material element $\mathscr{M}_{c}$. Let us put

$$
\Gamma:=\left\{(A, \alpha) \in \operatorname{Sym}_{0} \times \mathbb{R} \mid\|A\| \leq \alpha\right\} .
$$

Proposition 4.4. A state function $\psi: \Sigma \rightarrow \mathbb{R}$ is a normalized free-energy function for $\mathscr{M}_{c}$ if and only if, for each $\sigma=\left(E, E^{p}, \zeta\right) \in \Sigma$,

$$
\psi\left(E, E^{p}, \zeta\right)=\underline{\psi}\left(E, E^{p}, \zeta\right)-\varphi\left(E^{p}, \zeta\right),
$$

where $\varphi: \Gamma \rightarrow \mathbb{R}$ has the following properties:

$$
\begin{gathered}
\varphi(0,0)=0 ; \\
\varphi\left(A_{2}, \alpha_{2}\right) \geq \varphi\left(A_{1}, \alpha_{1}\right),
\end{gathered}
$$

for each $\left(A_{1}, \alpha_{1}\right),\left(A_{2}, \alpha_{2}\right) \in \Gamma$ such that

$$
\alpha_{2}-\alpha_{1} \geq\left\|A_{2}-A_{1}\right\| \text {. }
$$

As a consequence, every function $\varphi$ with properties (4.6) and (4.7) is nonnegative.

Proof. Let us suppose $\psi$ is a normalized free-energy function for $\mathscr{M}_{c}$. Then, with a similar procedure to that used to prove Proposition 8.1 in [11], we prove the existence of a function $\gamma: \Gamma \rightarrow \mathbb{R}$, such that

$$
\psi\left(E, E^{p}, \zeta\right)=\left(E-E^{p}\right) \cdot \mathbb{S}\left[E-E^{p}\right] / 2+\gamma\left(E^{p}, \zeta\right) .
$$

Defining $\varphi$ by

$$
\varphi(A, \alpha)=-\gamma(A, \alpha)+\mu \eta\|A\|^{2}+2 \mu \hat{\omega}(\alpha),
$$


we have

$$
\begin{gathered}
\varphi(0,0)=0 \\
\psi=\underline{\psi}-\varphi .
\end{gathered}
$$

Since $\psi$ is a free-energy function, we deduce from (2.9)

$$
\psi\left(\pi^{f}\right)-\psi\left(\pi^{i}\right) \leq w(\pi)
$$

for each process $\pi$. By combining (4.12) with (4.2) and (4.11) 2 , we obtain $\varphi\left(\pi^{f}\right)-$ $\varphi\left(\pi^{i}\right) \geq 0$, from which we deduce (4.7), taking into account Proposition 3.1 .

Conversely, if $\varphi: \Gamma \rightarrow \mathbb{R}$ is a function satisfying conditions (4.6) and (4.7) and if $\psi$ is defined by (4.5), we have, in view of (4.1) and (4.2),

$$
\begin{aligned}
\psi\left(\pi^{f}\right)-\psi\left(\pi^{i}\right) & =\underline{\psi}\left(\pi^{f}\right)-\underline{\psi}\left(\pi^{i}\right)-\varphi\left(E_{2}^{p}, \zeta_{2}\right)+\varphi\left(E_{1}^{p}, \zeta_{1}\right) \\
& =w(\pi)-\varphi\left(E_{2}^{p}, \zeta_{2}\right)+\varphi\left(E_{1}^{p}, \zeta_{1}\right) \leq w(\pi),
\end{aligned}
$$

for each process $\pi$ such that $\pi^{i}=\left(E_{1}, E_{1}^{p}, \zeta_{1}\right), \pi^{f}=\left(E_{2}, E_{2}^{p}, \zeta_{2}\right)$. Hence $\psi$ is really a free energy function for $\mathscr{M}_{c}$.

Proposition 4.5. Let $\varphi: \Gamma \rightarrow \mathbb{R}$ be a continuously differentiable function. Then $\varphi$ satisfies condition (4.7) if and only if

$$
\left\|\partial_{A} \varphi(A, \alpha)\right\| \leq \partial_{\alpha} \varphi(A, \alpha) \text { for each }(A, \alpha) \in \Gamma .
$$

Proof. Let us suppose $\varphi$ satisfies condition (4.7). For $(A, \alpha) \in \Gamma$ and $B \in \operatorname{Sym}_{0}$, let us put

$$
\widehat{A}(\tau)=A+\tau B, \quad \hat{\alpha}(\tau)=\alpha+\tau\|B\|, \quad \tau \geq 0,
$$

so that we have

$$
\hat{\alpha}(\tau)-\hat{\alpha}(0)=\tau\|B\|=\|\widehat{A}(\tau)-\widehat{A}(0)\| .
$$

In view of (4.15), condition (4.7) implies

$$
\varphi(\widehat{A}(\tau), \hat{\alpha}(\tau)) \geq \varphi(\widehat{A}(0), \hat{\alpha}(0)),
$$

from which we obtain

$$
\partial_{A} \varphi(A, \alpha) \cdot B+\partial_{\alpha} \varphi(A, \alpha)\|B\| \geq 0 .
$$

Moreover, choosing $A_{1}=A_{2}$ and $\alpha_{2} \geq \alpha_{1} \geq\left\|A_{1}\right\|=\left\|A_{2}\right\|$ in (4.7), we establish that $\alpha \mapsto \varphi(A, \alpha)$ is a nondecreasing function, and, therefore, we have

$$
\partial_{\alpha} \varphi(A, \alpha) \geq 0 \text {. }
$$

On the other hand, by observing that $\partial_{A} \varphi(A, \alpha) \in \mathrm{Sym}_{0}$, it is easy to check that conditions (4.17) and (4.18) imply inequality (4.13).

Conversely, let us suppose that $\varphi$ satisfies condition (4.13). Choosing $\left(A_{1}, \alpha_{1}\right)$ and $\left(A_{2}, \alpha_{2}\right)$ in $\Gamma$ such that

$$
\alpha_{2} \geq \alpha_{1}+\left\|A_{2}-A_{1}\right\|
$$


by means of a procedure similar to the one used to prove Proposition 3.1, we prove the existence of a path $\tau \mapsto(\widehat{A}(\tau), \hat{\alpha}(\tau))$ on $[0,1]$, which takes its values in $\Gamma$, such that

$$
\widehat{A}(0)=A_{1} ; \quad \widehat{A}(1)=A_{2}
$$

and

$$
\hat{\alpha}(\tau)=\alpha_{1}+\int_{0}^{\tau}\left\|\dot{A}\left(\tau^{\prime}\right)\right\| d \tau^{\prime}
$$

Differentiating the function $\tau \mapsto \varphi(\widehat{A}(\tau), \hat{\alpha}(\tau))$ and observing that condition (4.13) implies that inequality (4.17) is verified for each $(A, \alpha) \in \Gamma$ and for each $B \in \operatorname{Sym}_{0}$, we obtain

$$
\begin{aligned}
\dot{\varphi}(\widehat{A}(\tau), \hat{\alpha}(\tau)) & =\partial_{A} \varphi(\widehat{A}(\tau), \hat{\alpha}(\tau)) \cdot \dot{A}(\tau)+\partial_{\alpha} \varphi(\widehat{A}(\tau), \hat{\alpha}(\tau)) \dot{\alpha}(\tau) \\
& =\partial_{A} \varphi(\widehat{A}(\tau), \hat{\alpha}(\tau)) \cdot \dot{A}(\tau)+\partial_{\alpha} \varphi(\hat{A}(\tau), \hat{\alpha}(\tau))\|\dot{A}(\tau)\| \geq 0,
\end{aligned}
$$

where the last step has been obtained in view of (4.21). Thus, bearing in mind (4.20), we get

$$
\varphi\left(A_{2}, \alpha_{2}\right)=\varphi(\widehat{A}(1), \hat{\zeta}(1)) \geq \varphi(\widehat{A}(0), \hat{\alpha}(0))=\varphi\left(A_{1}, \alpha_{1}\right) .
$$

For each process $\pi \in \Pi$ and $\tau \in\left[0, d_{\pi}[\right.$, we call

$$
\widehat{S}_{\pi}(\tau) \cdot \dot{E}_{\pi}^{p}(\tau)
$$

the plastic power. In view of (3.3) and (3.6) we have

$$
\widehat{S}_{\pi}(\tau) \cdot \dot{E}_{\pi}^{p}(\tau)=2 \mu\left(\widehat{E}_{\pi}(\tau)-\widehat{E}_{\pi}^{p}(\tau)\right) \cdot \dot{E}_{\pi}^{p}(\tau) .
$$

Thus, the plastic power is nonnegative for each process $\pi \in \Pi$ and $\tau \in\left[0, d_{\pi}[\right.$ if and only if

$$
\widehat{E}^{p}(\sigma) \in E(\sigma) \text { for each } \sigma \in \Sigma .
$$

On the other hand, we deduce from (3.14) that (4.23) is verified if and only if

$$
\eta\left\|\widehat{E}^{p}(\sigma)\right\| \leq \tilde{\rho}(\hat{\zeta}(\sigma)) \text { for each } \sigma \in \Sigma,
$$

i.e., in view of (3.3), if and only if

$$
\eta \leq \alpha^{-1} \tilde{\rho}(\alpha) \text { for each } \alpha>0 .
$$

In particular, for a material element with isotropic work-hardening the plastic power is nonnegative for each process $\pi \in \Pi$ and $\tau \in\left[0, d_{\pi}[\right.$.

The result proved by means of the following proposition has been obtained by Owen in a slightly different context [15].

Proposition 4.6. The state function

$$
\psi^{*}\left(E-E^{p}\right):=\left(E-E^{p}\right) \cdot \mathbb{S}\left[E-E^{p}\right] / 2
$$

is a normalized free-energy function for $\mathscr{M}_{c}$ if and only if the plastic power is nonnegative for each process $\pi \in \Pi$ and $\tau \in\left[0, d_{\pi}[\right.$.

Proof. In view of (4.5), we have

$$
\varphi^{*}\left(E^{p}, \zeta\right):=\underline{\psi}\left(E, E^{p}, \zeta\right)-\psi^{*}\left(E-E^{p}\right)=\mu \eta\left\|E^{p}\right\|^{2}+2 \mu \hat{\omega}(\zeta) .
$$


An easy computation proves that $\varphi^{*}$ satisfies condition (4.13) if and only if

$$
\eta\|A\| \leq \tilde{\rho}(\alpha) \text { for each }(A, \alpha) \in \Gamma .
$$

Then, the desired result follows from (4.24), (4.25), and Propositions 4.5 and 4.4.

The next Proposition 4.7 provides a few examples of free-energy functions for $\mathscr{M}_{c}$. For this purpose, let us consider the following functions, on $\Gamma$ :

$$
\varphi_{\beta}(A, \alpha):=2 \mu \beta \hat{\omega}(\alpha),
$$

where $\beta \geq 0$ is a given parameter;

$$
\begin{aligned}
& \varphi^{1}(A, \alpha):=2 \mu[\hat{\omega}(\alpha)-\hat{\omega}(\|A\|)] \\
& \varphi^{2}(A, \alpha):=2 \mu[\hat{\omega}(\alpha)+\hat{\omega}(\|A\|)] .
\end{aligned}
$$

Proposition 4.7. The functions

$$
\begin{aligned}
\psi_{\beta}\left(E, E^{p}, \zeta\right) & :=\underline{\psi}-\varphi_{\beta} \\
& =\left(E-E^{p}\right) \cdot \mathbb{S}\left[E-E^{p}\right] / 2+\mu \eta\left\|E^{p}\right\|^{2}+2 \mu(1-\beta) \hat{\omega}(\zeta), \\
\psi^{1}\left(E, E^{p}, \zeta\right) & :=\underline{\psi}-\varphi^{1} \\
& =\left(E-E^{p}\right) \cdot \mathbb{S}\left[E-E^{p}\right] / 2+\mu \eta\left\|E^{p}\right\|^{2}+2 \mu \hat{\omega}\left(\left\|E^{p}\right\|\right), \\
\psi^{2}\left(E, E^{p}, \zeta\right) & :=\underline{\psi}-\varphi^{2} \\
& =\left(E-E^{p}\right) \cdot \mathbb{S}\left[E-E^{p}\right] / 2+\mu \eta\left\|E^{p}\right\|^{2}-2 \mu \hat{\omega}\left(\left\|E^{p}\right\|\right)
\end{aligned}
$$

are normalized free-energy functions for $\mathscr{M}_{c}$.

Proof. The functions $\varphi_{\beta}, \varphi^{1}$, and $\varphi^{2}$, defined by relations (4.27), satisfy condition (4.6) and therefore, in view of Proposition 4.4, it remains to be proved that $\varphi_{\beta}, \varphi^{1}$, and $\varphi^{2}$ satisfy the hypotheses of Proposition 4.5 , too.

The function $\varphi_{\beta}$ is continuously differentiable on $\Gamma$ and, for each $(A, \alpha) \in \Gamma$, we have

$$
\partial_{\alpha} \varphi_{\beta}(A, \alpha)=2 \mu \beta \tilde{\rho}(\alpha) \geq 0=\left\|\partial_{A} \varphi_{\beta}(A, \alpha)\right\| .
$$

The functions $\varphi^{1}$ and $\varphi^{2}$ are continuously differentiable on the set

$$
\Gamma^{*}:=\{(A, \alpha) \in \Gamma \mid A \neq 0\}
$$

and, for each $(A, \alpha) \in \Gamma^{*}$, we get

$$
\left\|\partial_{A} \varphi^{1}(A, \alpha)\right\|=\left\|\partial_{A} \varphi^{2}(A, \alpha)\right\|=2 \mu \tilde{\rho}(\|A\|) .
$$

Therefore, we can put

$$
\left\|\partial_{A} \varphi^{1}(0, \alpha)\right\|=\left\|\partial_{A} \varphi^{2}(0, \alpha)\right\|=2 \mu \tilde{\rho}(0) .
$$

Moreover, since, for $(A, \alpha) \in \Gamma$, we have

$$
\partial_{\alpha} \varphi^{1}(A, \alpha)=\partial_{\alpha} \varphi^{2}(A, \alpha)=2 \mu \tilde{\rho}(\alpha),
$$

the desired result follows from the fact that $\tilde{\rho}$ is an increasing function.

With Proposition 4.3 we have proved that the set of free-energy functions corresponding to $\mathscr{M}_{c}$ has an upper bound. That this set has no lower bound is proved by the next proposition. 
Proposition 4.8. For each state $\sigma=\left(E, E^{p}, \zeta\right)$ with $\zeta \neq 0$, and for each $\gamma>0$, there exists $\beta>0$, such that the free-energy function $\psi_{\beta}$ defined by (4.28) ${ }_{1}$ satisfies $\psi_{\beta}(\sigma)<-\gamma$.

Proof. In view of the first statement of Proposition 4.7 it is enough to choose $\beta$ such that

$$
\beta>1+(2 \mu \hat{\omega}(\zeta))^{-1}\left(\gamma+\frac{1}{2}\left(E-E^{p}\right) \cdot \mathbb{S}\left[E-E^{p}\right]+\mu \eta\left\|E^{p}\right\|^{2}\right) .
$$

At the end of this subsection, let us calculate the work done by the exterior on $\mathscr{M}_{c}$, during a particular process that starts at the annealed state and whose strain and plastic strain paths are straight line segments in $\mathrm{Sym}_{0}$.

Let $\zeta_{1}>0, \tau_{0}>0$, and $M \in \operatorname{Sym}_{0}$, with $\|M\|=1$. Consider the mapping

$$
\pi=\left(\widehat{E}_{\pi}, \widehat{E}_{\pi}^{p}, \hat{\zeta}_{\pi}\right):\left[0,1+\tau_{0}\right] \rightarrow \operatorname{Sym}_{0} \times \operatorname{Sym}_{0} \times \mathbb{R}^{+},
$$

where

$$
\begin{gathered}
\widehat{E}_{\pi}(\tau)=\left\{\begin{aligned}
&\left(\tau / \tau_{0}\right) \tilde{\rho}(0) M \text { for } \tau \in\left[0, \tau_{0}\right], \\
& {\left[\zeta_{1}\left(\tau-\tau_{0}\right)(1+\eta)+\tilde{\rho}\left(\zeta_{1}\left(\tau-\tau_{0}\right)\right)\right] M \text { for } \tau \in\left[\tau_{0}, 1+\tau_{0}\right] }
\end{aligned}\right. \\
\widehat{E}_{\pi}^{p}(\tau)= \begin{cases}0 \text { for } \tau \in\left[0, \tau_{0}\right], \\
\zeta_{1}\left(\tau-\tau_{0}\right) M \text { for } \tau \in\left[\tau_{0}, 1+\tau_{0}\right]\end{cases} \\
\zeta_{\pi}(\tau)= \begin{cases}0 \text { for } \tau \in\left[0, \tau_{0}\right], \\
\zeta_{1}\left(\tau-\tau_{0}\right) & \text { for } \tau \in\left[\tau_{0}, 1+\tau_{0}\right] .\end{cases}
\end{gathered}
$$

It can be checked from (3.8)-(3.12) that $\pi$ is a process that starts at the annealed state $\sigma_{0}$ and ends at

$$
\pi^{f}=\left(\left[\zeta_{1}(1+\eta)+\tilde{\rho}\left(\zeta_{1}\right)\right] M, \zeta_{1} M, \zeta_{1}\right) .
$$

The value of work $w(\pi)$ can be deduced from (4.1), (4.2), and (4.35):

$$
w(\pi)=\mu\left(\eta \zeta_{1}+\tilde{\rho}\left(\zeta_{1}\right)\right)^{2}+\mu \eta\left(\zeta_{1}\right)^{2}+2 \mu \hat{\omega}\left(\zeta_{1}\right) .
$$

In Fig. 1 (see p. 316), which plots the stress norm versus the strain norm, the work $w(\pi)$ is represented by the area of opqe; the first two terms on the right-hand side of (4.36) are represented by the areas of triangles rqe and pts, respectively, and the third term is represented by the sum of the areas of pqt and opsr.

4.2. The material element $\mathscr{M}_{k}$. The proofs of the following two propositions are similar to those of the second step of Proposition 3.1 and of Proposition 4.2, and have therefore been omitted.

Proposition 4.9. The material element $\mathscr{M}_{k}$ satisfies the condition of perfect accessibility.

Proposition 4.10. Let $\sigma_{1}=\left(E_{1}, E_{1}^{p}\right)$ and $\sigma_{2}=\left(E_{2}, E_{2}^{p}\right)$ be two states and let $\pi$ be a process such that $\pi^{i}=\sigma_{1}$ and $\pi^{f}=\sigma_{2}$. Then, we have

$$
\begin{aligned}
w(\pi)= & \left\{\left(E_{2}-E_{2}^{p}\right) \cdot \mathbb{S}\left[E_{2}-E_{2}^{p}\right]-\left(E_{1}-E_{1}^{p}\right) \cdot \mathbb{S}\left[E_{1}-E_{1}^{p}\right]\right\} / 2 \\
& +\mu \eta\left\{\left\|E_{2}^{p}\right\|^{2}-\left\|E_{1}^{p}\right\|^{2}\right\}+2 \mu \rho_{0} \zeta(\pi),
\end{aligned}
$$




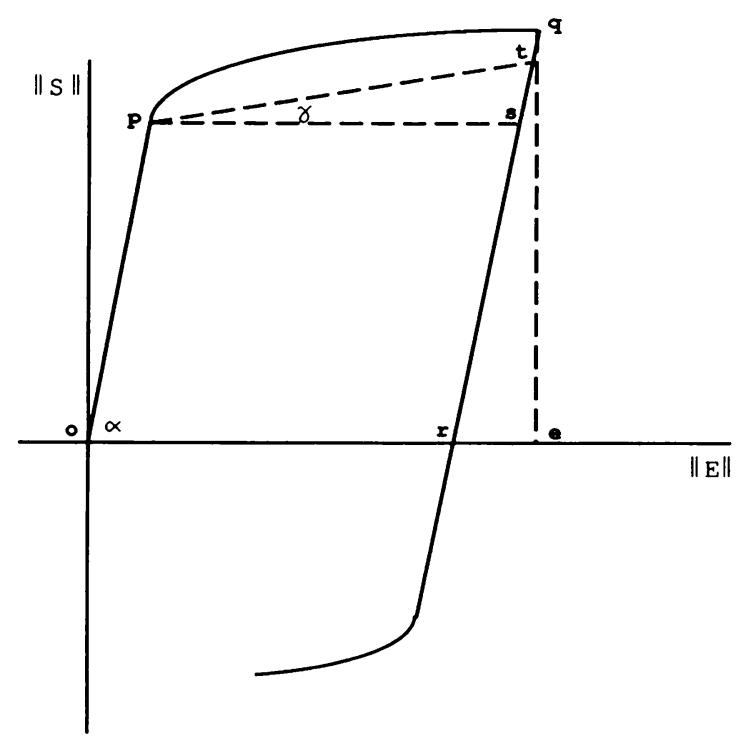

FIG. 1. $\|S\|$ VERSUS $\|E\| . \alpha=\operatorname{tg}^{-1}(2 \mu) ; \gamma=t g^{-1}(2 \mu \eta /(1+\eta))$.

where

$$
\zeta(\pi):=\int_{0}^{d_{\pi}}\left\|\dot{E}_{\pi}^{p}(\tau)\right\| d \tau .
$$

Unlike the case of materials that harden in a combined way, the work $w(\pi)$ now depends not only on $\pi^{i}$ and $\pi^{f}$, but on the entire process $\pi$ because of the term $2 \mu \rho_{0} \zeta(\pi)$

Let $\sigma_{0}:=(0,0)$ be the annealed state of $\mathscr{M}_{k}$; a free-energy function $\psi$ for $\mathscr{M}_{k}$ is said to be normalized if $\psi\left(\sigma_{0}\right)=0$.

The following Propositions 4.11 and 4.12 characterize the free-energy functions corresponding to $\mathscr{M}_{k}$ [16].

Proposition 4.11. A state function $\psi: \Sigma \rightarrow \mathbb{R}$ is a normalized free-energy function for $\mathscr{M}_{k}$ if and only if, for each $\sigma=\left(E, E^{p}\right) \in \Sigma$,

$$
\psi\left(E, E^{p}\right)=\psi^{*}\left(E-E^{p}\right)+\tilde{\gamma}\left(E^{p}\right),
$$

where $\psi^{*}$ is the function defined by means of relation (4.26), and $\tilde{\gamma}: \operatorname{Sym}_{0} \rightarrow \mathbb{R}$ has the two following properties:

$$
\begin{gathered}
\tilde{\gamma}(0)=0 \\
\tilde{\gamma}\left(A_{2}\right)-\tilde{\gamma}\left(A_{1}\right) \leq \mu \eta\left(\left\|A_{2}\right\|^{2}-\left\|A_{1}\right\|^{2}\right)+2 \mu \rho_{0}\left\|A_{2}-A_{1}\right\|
\end{gathered}
$$

for each $A_{1}, A_{2} \in \mathrm{Sym}_{0}$.

Proof. Let us suppose $\psi$ is a normalized free-energy function for $\mathscr{M}_{k}$. With a similar procedure to the one used to prove Proposition 8.1 in [11], we can prove the existence of a function $\tilde{\gamma}: \operatorname{Sym}_{0} \rightarrow \mathbb{R}$ that satisfies (4.39) and (4.40). Let $\sigma_{1}=$ $\left(E_{1}, E_{1}^{p}\right)$ and $\sigma_{2}=\left(E_{2}, E_{2}^{p}\right)$ be any two states and $\pi$ the special process such that

$$
\pi^{i}=\sigma_{1}, \quad \pi^{f}=\sigma_{2} ; \quad\left\|E_{2}^{p}-E_{1}^{p}\right\|=\zeta(\pi)
$$


the existence of which is proved as the third step of Proposition 3.1. From (4.37) we deduce

$$
w(\pi)=\psi^{*}\left(\sigma_{2}\right)-\psi^{*}\left(\sigma_{1}\right)+\mu \eta\left(\left\|E_{2}^{p}\right\|^{2}-\left\|E_{1}^{p}\right\|^{2}\right)+2 \mu \rho_{0}\left\|E_{2}^{p}-E_{1}^{p}\right\|
$$

and, together with (2.9), this implies (4.41).

Conversely, let us suppose $\psi$ is defined by relation (4.39), with $\tilde{\gamma}$ satisfying (4.40) and (4.41). We then get

$$
\begin{aligned}
\psi\left(\sigma_{2}\right)-\psi\left(\sigma_{1}\right) & \leq \psi^{*}\left(\sigma_{2}\right)-\psi^{*}\left(\sigma_{1}\right)+\mu \eta\left(\left\|E_{2}^{p}\right\|^{2}-\left\|E_{1}^{p}\right\|^{2}\right)+2 \mu \rho_{0} \zeta(\pi) \\
& =w(\pi)
\end{aligned}
$$

which, in view of (2.9), proves that $\psi$ is a free-energy function for $\mathscr{M}_{k}$.

Proposition 4.12. Let $\tilde{\gamma}: \operatorname{Sym}_{0} \rightarrow \mathbb{R}$ be a continuously differentiable function. Then, $\tilde{\gamma}$ satisfies condition (4.41) if, and only if, we get

$$
\left\|\partial_{A} \tilde{\gamma}-2 \mu \eta A\right\| \leq 2 \mu \rho_{0} \text { for each } A \in \operatorname{Sym}_{0} .
$$

Proof. Let us suppose $\psi$ satisfies condition (4.41). For $A, B \in \operatorname{Sym}_{0}$ with $\|B\|=1$, let

$$
\widehat{A}(\tau):=A+\tau B, \quad \tau \geq 0 .
$$

Given

$$
\xi(\tau):=\tilde{\gamma}(\widehat{A}(\tau))-\tilde{\gamma}(\widehat{A}(0))-\mu \eta\left(\tau^{2}+2 \tau A \cdot B\right)-2 \mu \rho_{0} \tau,
$$

from (4.41) and (4.47) we deduce $\xi(0)=0 ; \xi(\tau) \leq 0$ for $\tau \geq 0$, from which we obtain

$$
\left(\partial_{A} \tilde{\gamma}-2 \mu \eta A\right) \cdot B \leq 2 \mu \rho_{0}
$$

which, in view of the arbitrariness of $B$, implies (4.45).

Conversely, let us suppose $\tilde{\gamma}$ satisfies (4.45) so that, for each $A, B \in \operatorname{Sym}_{0},(4.48)$ proves to be satisfied. Let $\sigma_{1}=\left(E_{1}, E_{1}^{p}\right)$ and $\sigma_{2}=\left(E_{2}, E_{2}^{p}\right)$ be two states and let $\pi$ be any process such that $\pi^{i}=\sigma_{1}, \pi^{f}=\sigma_{2}$. In view of (4.48), (3.37), and (4.38), we get

$$
\begin{aligned}
\tilde{\gamma}\left(E_{2}^{p}\right)-\tilde{\gamma}\left(E_{1}^{p}\right) & =\int_{0}^{d_{\pi}} \dot{\gamma}(\tau) d \tau=\int_{0}^{d_{\pi}} \partial_{E^{p}} \tilde{\gamma}\left(E^{p}(\tau)\right) \cdot \dot{E}^{p}(\tau) d \tau \\
& \leq \int_{0}^{d_{\pi}}\left(2 \mu \eta E^{p}(\tau) \cdot \dot{E}^{p}(\tau)+2 \mu \rho_{0} \dot{\zeta}(\tau)\right) d \tau \\
& =\mu \eta\left(\left\|E_{2}^{p}\right\|^{2}-\left\|E_{1}^{p}\right\|^{2}\right)+2 \mu \rho_{0} \int_{0}^{d_{\pi}} \dot{\zeta}(\tau) d \tau \\
& \leq \mu \eta\left(\left\|E_{2}^{p}\right\|^{2}-\left\|E_{1}^{p}\right\|^{2}\right)+2 \mu \rho_{0}\left\|E_{2}^{p}-E_{1}^{p}\right\| .
\end{aligned}
$$

Therefore, (4.41) is satisfied.

The following proposition, which parallels Proposition 4.6, is an immediate consequence of (4.45) and Proposition 4.11. 
Proposition 4.13. The state function $\psi^{*}$ is a free-energy function for $\mathscr{M}_{k}$ if, and only if, $\mathscr{M}_{k}$ is an ideally plastic material element.

Since $\mathscr{M}_{k}$ is a material element with perfect accessibility, Proposition 2.4 guarantees that the set of the normalized free-energy functions has a maximum and a minimum; with the next proposition these are determined. Let $\psi(\sigma)$ and $\bar{\psi}(\sigma)$ be the two free-energy functions defined by relations (2.11) and (2.15), respectively, with $\sigma_{0}$ the annealed state of $\mathscr{M}_{k}$.

Proposition 4.14. We have

$$
\begin{aligned}
& \underline{\psi}(\sigma)=\left(E-E^{p}\right) \cdot \mathbb{S}\left[E-E^{p}\right] / 2+\mu \eta\left\|E^{p}\right\|^{2}+2 \mu \rho_{0}\left\|E^{p}\right\|, \\
& \bar{\psi}(\sigma)=\left(E-E^{p}\right) \cdot \mathbb{S}\left[E-E^{p}\right] / 2+\mu \eta\left\|E^{p}\right\|^{2}-2 \mu \rho_{0}\left\|E^{p}\right\| .
\end{aligned}
$$

Proof. From (2.11) and (4.37) we deduce, for each state $\sigma$,

$$
\underline{\psi}(\sigma)=\left(E-E^{p}\right) \cdot \mathbb{S}\left[E-E^{p}\right] / 2+\mu \eta\left\|E^{p}\right\|^{2}+2 \mu \rho_{0} \inf \left\{\zeta(\pi) \mid \pi^{i}=\sigma_{0}, \pi^{f}=\sigma\right\} .
$$

In view of (4.38), $\zeta(\pi)$ takes the minimum value when $\pi$ satisfies relation $(4.42)_{2}$ and this implies (4.49). Relation (4.50) is proved in a similar way.

Acknowledgments. I am grateful to M. Silhavy for a number of important suggestions. In particular, he is to be heartily thanked for providing the proof of Proposition 4.4 .

\section{REFERENCES}

[1] M. Lucchesi, D. R. Owen, and P. Podio-Guidugli, Materials with elastic range: A theory with a view toward applications. Part III, Arch. Rational Mech. Anal. 117, 53-96 (1992)

[2] M. Lucchesi and P. Podio-Guidugli, Materials with elastic range: $A$ theory with a view toward applications. Part I, Arch. Rational Mech. Anal. 102, 23-43 (1988)

[3] M. Lucchesi and P. Podio-Guidugli, Materials with elastic range: A theory with a view toward applications. Part II, Arch. Rational Mech. Anal. 110, 9-42 (1990)

[4] M. Šilhavý, On measures, convex cones, and foundations of thermodynamics. I. Systems with vectorvalued actions. II. Thermodynamic systems, Czechoslovak J. Phys. B30, 841-961 (1980)

[5] M. Šilhavý, Foundations of continuum thermodynamics, New Perspectives in Thermodynamics (J. Serrin, ed.), Springer, Berlin, 1986, pp. 33-48

[6] D. Owen, $A$ first course in the mathematical foundations of thermodynamics, Springer-Verlag, Berlin and New York, 1984

[7] B. Coleman and D. R. Owen, On the thermodynamics of semi-systems with restrictions on the accessibility of states, Arch. Rational Mech. Anal. 66, 173-181 (1977)

[8] M. Šilhavý, Lectures on the theory of plasticity, CNUCE, Mimeographed notes, Pisa, 1986

[9] M. Šilhavý and J. Kratochvil, A theory of inelastic behavior of materials, Part I. Ideal materials, Arch. Rational Mech. Anal. 65, 97-129 (1977)

[10] J. Kratochvil and M. Šilhavý, A theory of inelastic behavior of materials, Part II. Inelastic materials, Arch. Rational Mech. Anal. 65, 131-152 (1977)

[11] M. Lucchesi and M. Šilhavý, It yushin's conditions in non-isothermal plasticity, Arch. Rational Mech. Anal. 113, 121-163 (1991)

[12] E. Melan, Zur Plastizitat des raumlichen Kontinuums, Ing. Arch. 9, 116-126 (1938)

[13] R. Hill, The mathematical theory of plasticity, Oxford Univ. Press, Oxford, 1950

[14] M. Lucchesi, Esistenza e unicita' dello sforzo per materiali idealmente plastici soggetti a deformazioni finite, Boll. Un. Mat. Ital. 4-B, 1-16 (1990)

[15] D. R. Owen, Thermodynamics of materials with elastic range, Arch. Rational Mech. Anal. 31, 91-112(1968)

[16] B. Coleman and D. R. Owen, On the thermodynamics of elastic-plastic materials with temperature dependent moduli and yield stresses, Arch. Rational Mech. Anal. 70, 339-354 (1979) 